Note

\title{
Illumination conditions at the Asteroid 4 Vesta: Implications for the presence of water ice
}

\author{
Timothy J. Stubbs ${ }^{\text {a,c,d,*, Yongli Wang }}{ }^{\text {b,c,d }}$ \\ ${ }^{a}$ Center for Research and Exploration in Space Science and Technology, University of Maryland, Baltimore County, MD 21250, USA \\ ${ }^{\mathrm{b}}$ Goddard Planetary Heliophysics Institute, University of Maryland, Baltimore County, MD 21228, USA \\ ${ }^{\mathrm{c}}$ NASA Goddard Space Flight Center, Greenbelt, MD 20771, USA \\ ${ }^{\mathrm{d}}$ NASA Lunar Science Institute, NASA Ames Research Center, Moffett Field, CA 94035, USA
}

\section{A R T I C L E I N F O}

\section{Article history:}

Received 26 July 2011

Revised 4 November 2011

Accepted 4 November 2011

Available online 16 November 2011

\section{Keywords:}

Asteroid Vesta

Asteroids, Surfaces

Ices

Solar radiation

Thermal histories

\begin{abstract}
A B S T R A C T
The mean illumination conditions and surface temperatures over one orbital period are calculated for the Asteroid 4 Vesta using a coarse digital elevation model produced from Hubble Space Telescope images. Even with the anticipated effects of finer-scale topography taken into account, it is unlikely that any significant permanently shadowed regions currently exist on Vesta due to its large axial tilt $\left(\approx 27^{\circ}\right)$. However, under present day conditions, it is predicted that about half of Vesta's surface has an average temperature of less than $145 \mathrm{~K}$, which, based on previous thermal modeling of main belt asteroids, suggests that water ice could survive in the top few meters of the vestal regolith on billion-year timescales.
\end{abstract}

(c) 2011 Elsevier Inc. All rights reserved.

\section{Introduction}

As with other airless bodies in the Solar System, the surface of Vesta is directly exposed to the full solar spectrum. The degree of solar illumination plays a major role in processes at the surface, including heating, electric charging, chemistry, and exospheric production. It is likely that illumination (surface temperature) controls the transport and deposition of volatiles at Vesta, as has been proposed at the Moon (e.g., see Sunshine et al., 2009; Paige et al., 2010). If there are permanently shadowed regions (PSRs) on Vesta - or at least regions that, on average, receive very little solar illumination - then they could act as cold traps for volatiles. Schorghofer (2008) estimates that water ice on main belt asteroids can persist within the top few meters of a dusty surface layer over billions of years, if the mean surface temperature is $\lesssim 145 \mathrm{~K}$.

We predict the mean illumination conditions and surface temperatures over a vestal year (1325 days) using a modified version of the Vesta digital elevation model (DEM) produced by Thomas et al. (1997b). This allows us to make an assessment of the ability of volatiles, in particular water ice, to survive in the vestal regolith under present day conditions. The general methodology employed here with a relatively coarse DEM could readily be applied to other airless bodies in the Solar System. Determining illumination and thermal conditions is particularly timely given the Dawn mission's arrival at Vesta (Russell et al., 2007), with a payload including the Gamma Ray and Neutron Detector (GRaND). The regions with mean surface temperatures $\lesssim 145 \mathrm{~K}$ predicted here could correlate with possible future detections of hydrogen-rich deposits by GRaND, which may be indicative of sub-surface water ice.

* Corresponding author. Address: NASA Goddard Space Flight Center, Mail Code 695, Greenbelt, MD 20771, USA.

E-mail addresses: Timothy.J.Stubbs@NASA.gov (T.J. Stubbs), Yongli.Wang@ NASA.gov (Y. Wang).

${ }^{1}$ NASA Goddard Space Flight Center, Mail Code 674, Greenbelt, MD 20771, USA.

\section{The orbital and shape characteristics of 4 Vesta}

Vesta is located in the inner main asteroid belt, and its current orbital characteristics are summarized in Table 1. Based on Hubble Space Telescope (HST) images acquired in 1994 and 1996, Vesta is known to be a triaxial ellipsoid with radii of 289,280 and $229 \pm 5 \mathrm{~km}$ with a spin pole solution of $\mathrm{RA}=301^{\circ}, \mathrm{Dec}=41^{\circ}, \pm 5^{\circ}$ (revised values from Thomas et al. (1997b)). This corresponds to a large axial tilt, or obliquity, of $27.2 \pm 5^{\circ}$, which is in good agreement with more recent studies (Li et al., 2011). Vesta has a prograde rotation with a relatively short spin period for an asteroid of $5.342 \mathrm{~h}$. Vesta is close to being an oblate spheroid, but for the enormous impact crater centered near its south pole with a diameter of $\sim 460 \mathrm{~km}$ (Thomas et al., 1997a).

For the shape of Vesta we use the $5 \times 5^{\circ}$ DEM based on HST images (Thomas et al., 1997b). The orbital information for Vesta is obtained from the Dawn SPICE kernel, which is currently limited to the 1900-2100 epoch. However, before this DEM could be used for predicting illumination conditions, some modifications were required in order to produce more realistic results. Firstly, we had to smooth out some irregularities in the DEM poleward of $80^{\circ} \mathrm{N} / \mathrm{S}$ that were clearly associated with the meridional convergence, as opposed to any real topographic features. We found that this could be effectively dealt with by simply smoothing the relevant data points on the original DEM, which was achieved by calculating an average radial distance using one data point either side in latitude $\left( \pm 5^{\circ}\right)$ and four points either side in longitude $\left( \pm 20^{\circ}\right)$. Secondly, the $5 \times 5^{\circ} \mathrm{DEM}$ is too coarse for producing realistic illumination predictions $(\approx 22.5 \mathrm{~km}$ at the equator); so we decided to interpolate the DEM to a $1 \times 1^{\circ}$ resolution $(\approx 4.5 \mathrm{~km})$ using the modified Shepard's method (Renka, 1988), which is a multivariate interpolation technique that employs an inverse distance weighting algorithm. To our knowledge, this is the best interpolation method for increasing the resolution of coarse DEMs, since it has the advantages of producing smooth results while still exactly matching the values of the original DEM. Using this modified DEM, we produced revised estimates for the mean radius, surface area, volume and mean density of Vesta, as detailed in Table 1. 
Table 1

Summary of the orbital, physical and thermal properties relevant to Vesta.

\begin{tabular}{|c|c|}
\hline Property & Value \\
\hline Aphelion $^{\mathrm{a}}$ & $2.572 \mathrm{AU}$ \\
\hline Perihelion $^{\mathrm{a}}$ & $2.151 \mathrm{AU}$ \\
\hline Eccentricity $^{\mathrm{a}}$ & 0.08890 \\
\hline Semi-major axis, ${ }^{\text {a }} D$ & $2.36158 \mathrm{AU}$ \\
\hline Inclination to ecliptic plane $\mathrm{a}^{\mathrm{a}}$ & $7.13404^{\circ}$ \\
\hline Orbital period $^{\mathrm{a}}$ & 1325 days ( 3.63 years) \\
\hline Precession period ${ }^{\mathrm{a}}$ & 81,730 years \\
\hline Spin period ${ }^{\mathrm{a}, \mathrm{b}}$ & $5.342 \mathrm{~h}$ \\
\hline Spin pole solution ${ }^{\mathrm{b}}$ & $\mathrm{RA}=301^{\circ}, \mathrm{Dec}=41^{\circ}, \pm 5^{\circ}$ \\
\hline Obliquity (axial tilt) ${ }^{\mathrm{b}, \mathrm{c}}$ & $27.2 \pm 5^{\circ}$ \\
\hline Triaxial ellipsoidal radii ${ }^{\mathrm{b}}$ & $289,280,229 \pm 5 \mathrm{~km}$ \\
\hline Mean radius ${ }^{\mathrm{d}}$ & $266.6 \pm 8.8 \mathrm{~km}$ \\
\hline Surface area ${ }^{d}$ & $8.79 \pm 0.26 \times 10^{5} \mathrm{~km}^{2}$ \\
\hline Volume $^{\mathrm{d}}$ & $7.81 \pm 0.26 \times 10^{7} \mathrm{~km}^{3}$ \\
\hline $\operatorname{Mass}^{\mathrm{e}}$ & $2.586 \pm 0.002 \times 10^{20} \mathrm{~kg}$ \\
\hline Average density ${ }^{\mathrm{d}, \mathrm{e}}$ & $3.31 \pm 0.11 \times 10^{3} \mathrm{~kg} \mathrm{~m}^{-3}$ \\
\hline Solar constant, $S_{0}$ & $1366 \mathrm{~W} \mathrm{~m}^{-2}$ \\
\hline Solar constant at Vesta, $S_{0} / D^{2}$ & $245 \mathrm{~W} \mathrm{~m}^{-2}$ \\
\hline Bond albedo, ${ }^{\mathrm{f}} A$ & 0.20 \\
\hline Thermal emissivity, ${ }^{\mathrm{f}} \varepsilon$ & 0.9 \\
\hline Diurnal skin depth, ${ }^{\mathrm{f}} \delta_{d}$ & $0.00192 \mathrm{~m}$ \\
\hline Seasonal skin depth, ${ }^{\mathrm{f}} \delta_{s}$ & $0.148 \mathrm{~m}$ \\
\hline $\begin{array}{l}\text { Mean nightside surface temperature (hybrid } \\
\text { model) }\end{array}$ & $80 \mathrm{~K}$ \\
\hline
\end{tabular}

a Bills and Nimmo (2011).

b Thomas et al. (1997b) revised values.

c Li et al. (2011).

d The modified Vesta DEM presented here.

e Kuzmanoski et al. (2010).

f Chamberlain et al. (2007) and references therein.

\section{Illumination model}

The illumination model used here has been optimized for speed, accuracy and versatility by combining the ray tracing approach (e.g., Bussey et al., 2010) with the "horizon method" described by Mazarico et al. (2011). Since we are just interested in the illumination conditions for this study, we only need to calculate: (1) the fraction of the solar disk visible from the surface (accounting for topography along the horizon); (2) the solar incidence angle (including the effect of local slope); and (3) the normalized solar flux, which is determined using the previous two parameters. Given the uncertainties in the DEM, we do not consider sunlight scattered from other surface elements, just direct solar illumination.

Similar to Mazarico et al. (2011), the first step is to generate a database containing the elevation of the horizon at 36 equally-spaced azimuthal directions for every point on the DEM. Using this database, the elevation and slope of the horizon can then be calculated very efficiently for any given azimuthal direction. The elevation and angular extent of the Sun is given by the SPICE kernels, which then allows the fraction of the solar disk visible from the surface to be calculated. The DEM surface normals are calculated by taking the cross product of adjacent grid points, which then permits the solar incidence angle to be determined. However, unlike Mazarico et al. (2011), we do not rely on gnomonic projection in our calculations; therefore, we are not restricted to the polar regions and are able to achieve global coverage. Our model has been validated by comparisons with illumination simulations of the lunar polar regions generated by Mazarico et al. (2011) using Lunar Orbiting Laser Altimeter (LOLA) DEMs.

In our simulations we consider the average illumination conditions over one vestal year, which should be indicative of conditions during at least the past few hundred thousand years (cf. Bills and Nimmo, 2011). We found that the normalized solar flux results were typically accurate to within $<0.01 \%$ when calculations were performed 72 times per rotation period at 360 locations along the orbital path (separated equally in time).

\section{Hybrid thermal model}

In order to use our illumination predictions to assess the ability of volatiles to survive in the vestal regolith, we need to employ a thermal model. Schorghofer (2008) made theoretical estimates of the loss rate of buried water ice from main belt asteroids. He proposed that water ice can survive within the top few meters of a dusty surface layer for billions of years, if the mean surface temperature $\langle T\rangle$ is $<145 \pm 10 \mathrm{~K}$. As part of his study, two end member thermal models were investigated: the fast-rotating or isothermal latitude model, which assumes infinite thermal inertia; and the non-rotating or standard thermal model, which assumes zero thermal inertia (see Schorghofer (2008), and references therein). When compared with a model that included a more realistic multi-layered treatment of thermal inertia, it was shown that neither of the end member models provided reasonable estimates for $\langle T\rangle$. Therefore, we employ a hybrid thermal model that uses the illumination predictions to estimate the mean dayside temperatures $\left\langle T_{d}\right\rangle$, and assumes a constant mean nightside temperature $\left\langle T_{n}\right\rangle$. The assumptions that the dayside is in thermal equilibrium with the incident solar flux, while the nightside temperature does not vary significantly, is consistent with thermal observations of other airless bodies (e.g., Paige et al., 2010). The mean dayside temperature for each surface element is determined using the Stefan-Boltzmann law, $\left\langle T_{d}\right\rangle^{4}=(1-A)\left(S_{0}\langle F\rangle / \varepsilon \sigma D^{2}\langle V\rangle\right)$; where $\sigma$ is the Stefan-Boltzmann constant, $\langle V\rangle$ is the average fraction of the solar disk visible from the surface, $\langle F\rangle$ is the average normalized solar flux scaled to the semi-major axis $D$ (see Table 1 for more details). The overall mean surface temperature for the hybrid model is then given by $\left\langle T_{h}\right\rangle=\langle V\rangle\left\langle T_{d}\right\rangle+(1-\langle V\rangle)\left\langle T_{n}\right\rangle$, which produces estimates that lie between the two end member models, as expected.

To verify this hybrid approach we compare with results from Schorghofer (2008) that indicated $\approx 20 \%$ of the surface area of a spherical body with Vesta's semi-major axis and obliquity would have $\langle T\rangle<145 \mathrm{~K}$, which matches our model if $\left\langle T_{n}\right\rangle=83 \mathrm{~K}$. In Schorghofer's calculations, the orbital and thermal characteristics used are broadly consistent with those expected for Vesta (e.g., see Table 1), apart from a lower Bond albedo of $A=0.05$ (used in the above comparison). In our study we use $A=0.2$ and $\left\langle T_{n}\right\rangle=80 \mathrm{~K}$ with the hybrid thermal model, which for a Vesta-like spherical body results in $\left\langle T_{h}\right\rangle<145 \mathrm{~K}$ over $\approx 40 \%$ of its surface. We estimate the $\left\langle T_{h}\right\rangle$ uncertainties to be typically $<10 \mathrm{~K}$. For context, these thermal characteristics for Vesta predict a subsolar surface temperature at the semi-major axis of $249 \mathrm{~K}$.

\section{Results}

Illumination and thermal modeling predictions are presented in planetocentric coordinates with west longitudes plotted to the right of the prime meridian in order to be consistent with earlier presentations of Vesta DEMs (Thomas et al., 1997a,b).

\subsection{Average illumination conditions over a vestal year}

We present global predictions for the average illumination conditions at Vesta during one vestal year, with particular attention paid to the southern polar region (Fig. 1). The top row shows the modified Vesta DEM $\left(1 \times 1^{\circ}\right.$ resolution), which clearly reveals the large topographic variation relative to Vesta's basic triaxial ellipsoid shape. The giant impact crater can be readily identified in the southern polar region with its central peak at $65^{\circ} \mathrm{S}, 104^{\circ} \mathrm{W}$ and the lowest point at $71^{\circ} \mathrm{S}, 111^{\circ} \mathrm{E}$. In contrast, the northern polar region appears to be relatively featureless.

The second row shows the mean solar visibility $\langle V\rangle$, which reveals that the solar disk is typically seen about half the time in the tropics (between the dotted lines), as expected for a diurnal variation. At mid- to far-northern latitudes, $\langle V\rangle$ tends to increase slightly, probably due to Vesta's irregular oblate shape. Conversely, in the southern hemisphere, the effects of the giant impact crater are clearly evident with many distinct regions of low $\langle V\rangle$. As expected, the point with the highest $\langle V\rangle(0.566)$ is located close to the central peak at $65^{\circ} \mathrm{S}, 100^{\circ} \mathrm{W}$; while the point with the lowest $\langle V\rangle(0.363)$ is close to the topographic low at $70^{\circ} \mathrm{S}, 108^{\circ} \mathrm{E}$. The third row shows the mean solar incidence angles $\langle I\rangle$, where the expected general trend of low $\langle I\rangle$ around the equator, which then increases toward the poles, is evident. Due to the prominence of the central peak, the $\langle I\rangle$ values are typically much lower there than for the rest of the southern polar region.

The maps of mean normalized solar flux $\langle F\rangle$ (fourth row) have been scaled to the semi-major axis, and so can be used directly with the hybrid thermal model. The highest $\langle F\rangle$ value (0.302) is predicted at the equator at $0^{\circ}, 4^{\circ} \mathrm{E}$; this is consistent with expectations since each surface element will be illuminated about half the time, and so combined with a simple cosine dependence for a spherical body gives a maximum value of $0.318(1 / \pi)$. The lowest $\langle F\rangle$ value $(0.145)$ is located within the giant crater at $73^{\circ} \mathrm{S}, 30^{\circ} \mathrm{E}$. In spite of this, the northern and southern hemispheres have roughly the same surface area receiving $\langle F\rangle<0.15$. As anticipated for a body with such a significant axial tilt, we do not identify any permanently shadowed or permanently illuminated regions on Vesta. However, we have not yet considered the effects of finer-scale topography (see Section 5.2).

Using the hybrid thermal model described in Section 4 we convert the mean illumination conditions to mean surface temperatures $\left\langle T_{h}\right\rangle$ (bottom row), which gives a range of values from 123.4 to $150.8 \mathrm{~K}$, with a global annual average of $142.7 \mathrm{~K}$. Based on our results, under present day conditions about $52 \%$ of Vesta's surface is cold enough for water ice to have persisted in the top few meters of the regolith for billions of years should it have been delivered there. The $\left\langle T_{h}\right\rangle=145 \mathrm{~K}$ contour is indicated by the gray solid lines.

\subsection{Influence of finer-scale topographic features on illumination and thermal conditions}

Since the original DEM used in this study has a resolution of $5 \times 5^{\circ}$, the modified version is still only able to really resolve topographic features with spatial scales of $\sim 100 \mathrm{~km}$. It is well known that topography with much smaller spatial scales than this can have a significant influence on illumination conditions (e.g., Mazarico et al., 2011). Therefore, to investigate this influence we convolved a 

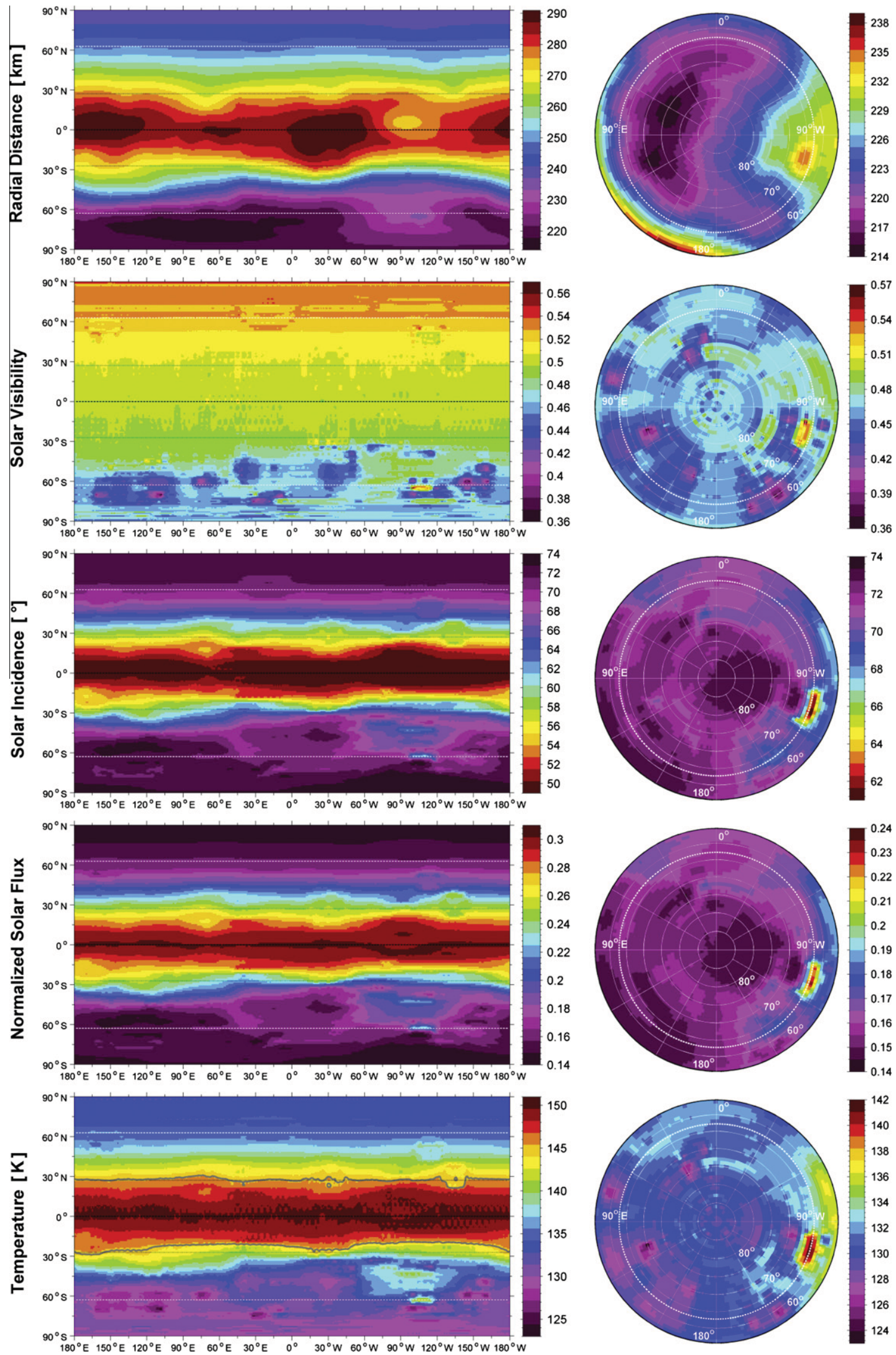

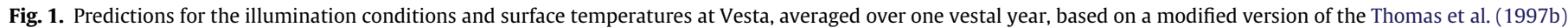

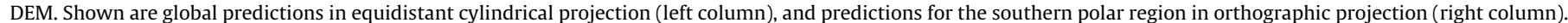

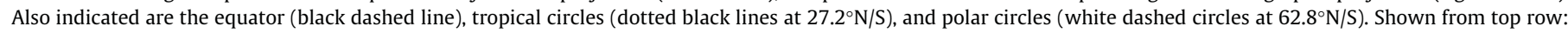
the radial planetocentric distance of the surface; the visible fraction of the solar disk; solar incidence angle; normalized solar flux (where 1 corresponds to the Sun directly

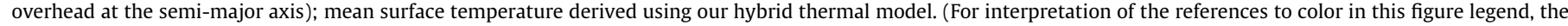
reader is referred to the web version of this article.) 

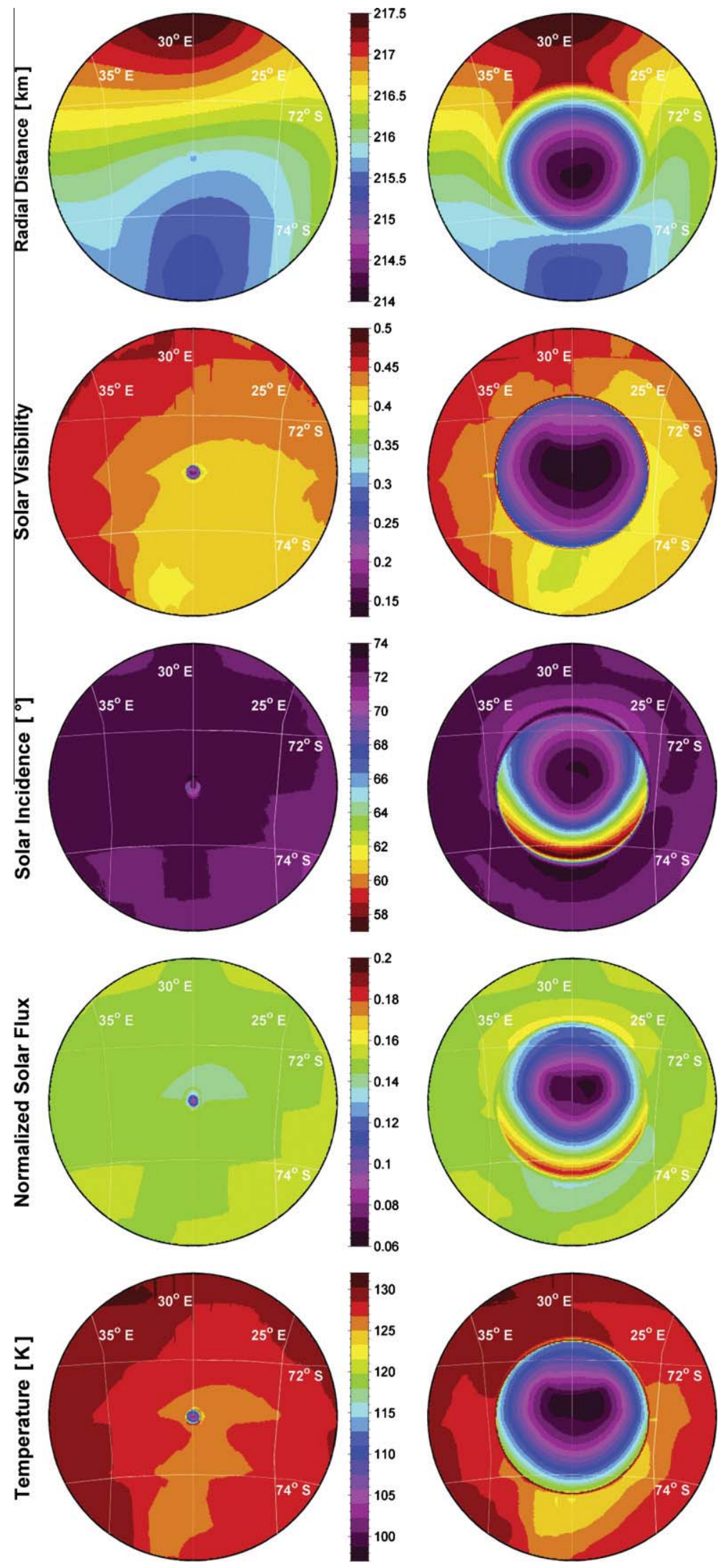

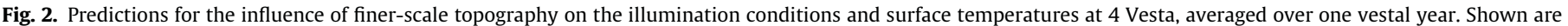

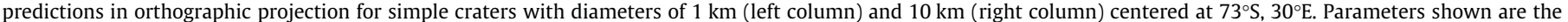
same as in Fig. 1. (For interpretation of the references to color in this figure legend, the reader is referred to the web version of this article.) 
simple crater shape with the modified DEM centered at $73^{\circ} \mathrm{S}, 30^{\circ} \mathrm{E}$, the location predicted to receive the minimum $\langle F\rangle$ in Section 5.1. In this study we use the typical shape characteristics for simple lunar craters with diameters $<15 \mathrm{~km}$, as described by Hörz et al. (1991) and references therein. In order to assess the dependence on the spatial scale of the craters, we investigate the illumination conditions and surface temperatures for a $1 \mathrm{~km}$ and a $10 \mathrm{~km}$ diameter crater averaged over one vestal year.

The results for the $1 \mathrm{~km}$ diameter crater are shown in the left column of Fig. 2 . Despite only producing an apparently minor perturbation to the local topography, this crater has a significant effect on average illumination conditions and surface temperatures. The values of $\langle F\rangle$ at the center of the crater floor drop by about a factor of 2 to $\approx 0.08$, which results in $\left\langle T_{h}\right\rangle$ values of only $\approx 101 \mathrm{~K}$. This is almost $30 \mathrm{~K}$ colder than if there were no crater present, and could result in water being thermally stable on the surface at that location for much of the vestal year (cf., Paige et al., 2010). The results for the $10 \mathrm{~km}$ diameter crater, shown in the right column of Fig. 2, reveal that this size crater produces a significant perturbation to the surrounding topography. In this example we can see how $\langle V\rangle$ values at the center of the crater decrease to $\approx 0.13$ (almost a factor of three drop), but increase along the narrow edge of the crater rim to $\sim 0.5$. Similar to the $1 \mathrm{~km}$ crater case, there is a significant decrease in $\langle I\rangle$ values on the equatorward facing southern crater wall of up to $\approx 15^{\circ}$. This suggests that scattered sunlight could sometimes play an important role in the thermal conditions within such a crater (cf. Paige et al., 2010). The values for $\langle F\rangle$ decrease to $\approx 0.065$ on the crater floor (more than a factor of two drop), while they increase to $\approx 0.18$ along a narrow crescent on the southern wall (up by $\approx 20 \%$ ) due to the decrease in $\langle I\rangle$ values discussed previously. The predicted change in $\left\langle T_{h}\right\rangle$ ranges from a decrease to $\approx 97 \mathrm{~K}$ on the crater floor, to an increase along the edge of the crater rim to $\approx 130 \mathrm{~K}$. Despite the increased $\langle F\rangle$, the southern crater wall is colder on average due to the decrease in $\langle V\rangle$. Conversely, the sharp increase in $\left\langle T_{h}\right\rangle$ along the edge of the crater rim is due to the increased $\langle V\rangle$.

Neither a $1 \mathrm{~km}$ nor a $10 \mathrm{~km}$ diameter crater is able to produce a PSR at this location, although the latter gets very close. In both cases there is a large perturbation to the average illumination conditions that produces an overall decrease in $\left\langle T_{h}\right\rangle$, which is anticipated to significantly affect the thermal stability of volatiles at the surface, as well as those buried in the regolith.

\section{Discussion and conclusions}

The depth to which the solar thermal perturbation can penetrate into the regolith can be assessed using the thermal skin depth $\delta$, which is dependent on the time period of the temperature oscillation (see Chamberlain et al. (2007), and references therein). Since Vesta has an obliquity of $\approx 27.2^{\circ}$, we must consider both the diurnal skin depth $\delta_{d}$ (rotation period), and the seasonal skin depth $\delta_{s}$ (orbital period). The $\delta_{d}$ value is relevant to equatorial and mid-latitude regions; while $\delta_{s}$ is applicable to the polar regions, which can be exposed to near-constant solar illumination during their summer seasons (see Table 1). If water ice does exist in the vestal regolith, then it will likely be covered by a desiccated layer of at least a few $\delta$ in thickness (cf. Chamberlain et al., 2007). Therefore, in the polar regions of Vesta it seems reasonable to expect any significant abundance of water ice to be buried at least a few tens of $\mathrm{cm}$ below the surface.

Recent observations of a $3 \mu \mathrm{m}$ absorption feature on the Moon with band depths of up to $\approx 15 \%$ indicate that its entire surface is hydrated during some portion of a lunation, with diurnal variations and other characteristics possibly consistent with hydroxyl formed by the interaction between solar wind protons and oxygen-bearing minerals (e.g., Sunshine et al., 2009). The $3 \mu \mathrm{m}$ absorption feature has also been observed on Vesta (Hasegawa et al., 2003), but the band depth was only $\approx 1 \%$ which seemed too weak to be consistent with the above solar wind implantation hypothesis. However, the apparent lack of space weathering on Vesta suggests that it is sufficiently magnetized to be able to shield most of its surface from solar wind protons (e.g., Vernazza et al., 2006). This proposed proton-shielding could also be the reason for the relatively weak $3 \mu \mathrm{m}$ absorption feature, and be indicative of lower than expected abundances of hydrated and/or hydroxylated minerals on the surface of Vesta.

Based on our illumination and thermal predictions for present day conditions, it is possible that water ice could have survived in the top few meters of the regolith in the polar regions of Vesta for billions of years (over about half of Vesta's surface) should it have been delivered there. As expected, topography plays an important role in determining the average illumination conditions and surface temperatures. In particular, small scale topographic features, such as $\sim 1-10 \mathrm{~km}$ diameter craters, can have a significant effect, and perhaps even permit water ice to be thermally stable at the surface $(\lesssim 100 \mathrm{~K}$ ) in some polar locations for much of the vestal year.

\section{Acknowledgments}

The development of the illumination code, and its application to Vesta, were funded by NASA Grants NNX08AM76G and NNX09AG78A, respectively. The NASA Planetary Data System (PDS) facilitated access to the 4 Vesta DEM used in this investigation.

\section{References}

Bills, B.G., Nimmo, F., 2011. Forced obliquities and moments of inertia of Ceres and Vesta. Icarus 213 (2), 496-509.

Bussey, D.B. et al., 2010. Illumination conditions of the south pole of the Moon derived using Kaguya topography. Icarus 208, 558-564.

Chamberlain, M.A. et al., 2007. Submillimeter lightcurves of Vesta. Icarus 192, 448459.

Hasegawa, S. et al., 2003. Evidence of hydrated and/or hydroxylated minerals on the surface of Asteroid 4 Vesta. Geophys. Res. Lett. 30 (21), 2123.

Hörz, F. et al., 1991. Lunar surface processes. Lunar Source Book: A User's Guide to the Moon. Cambridge University Press, pp. 61-120.

Kuzmanoski, M. et al., 2010. The mass of (4) Vesta derived from its largest gravitational effects. Astron. J. 140 (3), 880-886.

Li, J.-Y. et al., 2011. Improved measurement of Asteroid (4) Vesta's rotational axis orientation. Icarus 211, 528-534.

Mazarico, E. et al., 2011. Illumination conditions of the lunar polar regions using LOLA topography. Icarus 211, 1066-1081.

Paige, D.A. et al., 2010. Diviner lunar radiometer observations of cold traps in the Moon's south polar region. Science 330, 479-482.

Renka, R.J., 1988. Multivariate interpolation of large sets of scattered data. ACM Trans. Math. Softw. 14 (2), 139-148.

Russell, C.T. et al., 2007. Dawn mission to Vesta and Ceres. Earth Moon Planets 101, 65-91.

Schorghofer, N., 2008. The lifetime of ice on main belt asteroids. Astrophys. J. 682, 697-705.

Sunshine, J.M. et al., 2009. Temporal and spatial variability of lunar hydration as observed by the Deep Impact Spacecraft. Science 326, 565-568.

Thomas, P.C. et al., 1997a. Impact excavation on Asteroid 4 Vesta: Hubble Space Telescope results. Science 277, 1492-1495.

Thomas, P.C. et al., 1997b. Vesta: Spin pole, size and shape from HST images. Icarus $128,88-94$

Vernazza, P. et al., 2006. Asteroid colors: A novel tool for magnetic field detection? The case of Vesta. Astron. Astrophys. 451 (3), L43-L46. 